\title{
The Temporal Dynamics of Cortical Normalization Models of Decision-making
}

\author{
Thomas LoFaro ${ }^{1,}$, Kenway Louie ${ }^{2}$, Ryan Webb ${ }^{3}$, Paul W. Glimcher ${ }^{2}$
}

\begin{abstract}
Normalization is a widespread neural computation in both early sensory coding and higher-order processes such as attention and multisensory integration. It has been shown that during decision-making, normalization implements a context-dependent value code in parietal cortex. In this paper we develop a simple differential equations model based on presumed neural circuitry that implements normalization at equilibrium and predicts specific time-varying properties of value coding. Moreover, we show that when parameters representing value are changed, the solution curves change in a manner consistent with normalization theory and experiment. We show that these dynamic normalization models naturally implement a time-discounted normalization over past activity, implying an intrinsic reference-dependence in value coding of a kind seen experimentally. These results suggest that a single network mechanism can explain transient and sustained decision activity, reference dependence through time discounting, and hence emphasizes the importance of a dynamic rather than static view of divisive normalization in neural coding.
\end{abstract}

Keywords: neuroscience, neuroeconomics, cortical normalization, differential equations

\section{Introduction}

Neuroeconomics is a multidisciplinary field where techniques and ideas from neuroscience, psychology, economics, and mathematics are applied to develop an understanding of the neurobiological mechanisms of decision-making. While neuroeconomic research takes many forms, we focus on value-guided decision-making at the level of a small network of neurons.

Like most neurons in visually-responsive brain regions, neurons in the monkey lateral intraparietal area (LIP) respond selectively to visual stimuli in a circumscribed region of visual space termed the response field (RF). However, LIP neurons also show motor-related activity specific for eye movements to the response field, suggesting a role in the sensory-tomotor transformation that underlies the decision process. Consistent with decision-related sensorimotor integration, neural activity in LIP (measured in spikes per second) is neither purely sensory nor purely motor, but instead reflects an integrated decision variable representing the 'value' of the eye movement towards the response field of the neuron $[5,8,9]$. We have recently shown that this value code is computed relative to the value of alternative options via a mechanism known as divisive normalization [6]. The idea behind this mechanism is similar to the normalization of a vector $\vec{v}$, with the response of a given neuron (an

\footnotetext{
${ }^{1}$ Department of Mathematics and Computer Science, Gustavus Adolphus College

${ }^{2}$ Center for Neural Science, New York University and Institute for the Interdisciplinary Study of Decision Making, New York University

${ }^{3}$ Rotman School of Management, University of Toronto

* Correspondence: tlofaro@gustavus.edu
} 
entry in $\vec{v}$ ) divided by the summed activity of a larger neuronal pool (the norm of $\vec{v}$ ) [10]. Normalization models have been successfully applied to a wide variety neural phenomena in multiple levels and modalities of sensory processing [2].

Current models of normalization, and value coding by the nervous system more generally, are often characterized in a static manner over long intervals of "delay period" activity. However from an empirical standpoint, neural activity displays significant time-varying changes over the choice process that have not been well addressed. The discovery of normalization in LIP offers a link between value representation, potential circuit mechanisms, and neural dynamics. In [7] we propose a possible network mechanism that implements normalization in LIP during value-related decision tasks. This model is described by a system of differential equations and we show that it

- implements divisive normalization at equilibrium and qualitatively describes the equilibrium behavior previously shown in [6],

- agrees with observed temporal dynamics during decision-making tasks as illustrated in the left panels of Figure 1, and

- describes both temporal and equilibrium changes in firing rates in response to reward value as illustrated in the right panels of Figure 1.
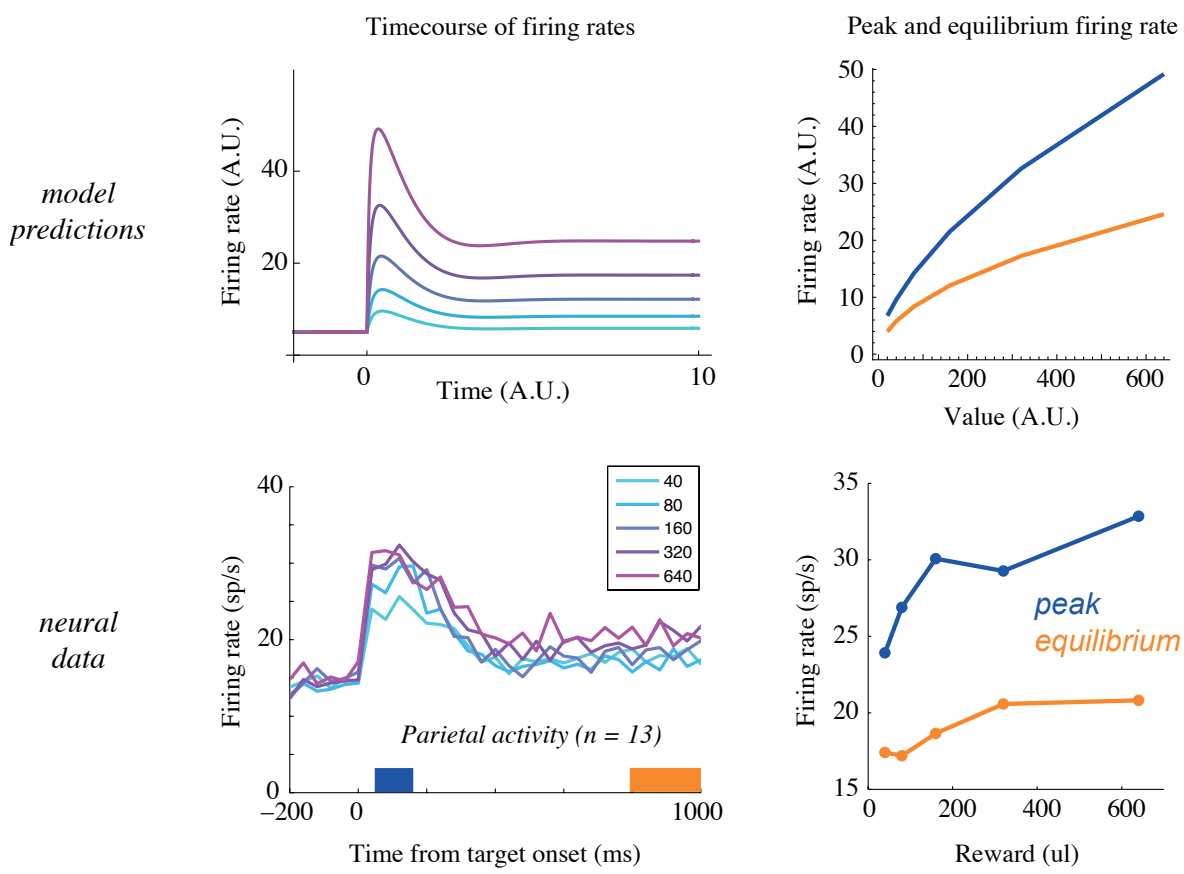

Figure 1: A comparison of model solutions and neural data from [7]. Comparison of model (top) and experimental (bottom) firing rate timecourses during reward tasks. The experimental reward value (in microliters) and model value parameter $(V)$ are indicated in the legend. On the right is a comparison of peak (blue) and equilibrium (orange) firing rates as a function of reward value in model (top) and experiment (bottom). Maximum firing rates for the model solutions were approximated numerically using the Mathematica [12] FindMaximum function. Equations (1) were solved with $N=1$ and initial conditions of $G(0)=0, R(0)=5$ for both model figures. Maximum and equilibrium firing rates in experiment were determined by averaging firing rates in color coded intervals indicated on the bottom left panel.

In what follows we provide the general mathematical foundations for the special-case used in [7]. That paper used a simplified dynamic normalization model and focused primarily 
on single-choice and two-choice models and experiments. Here we consider a wider range of dynamic normalization models that better reflect the range of non-dynamic models used in practice. In Section 2 we develop the generalized dynamic normalization model with $N$ alternatives from assumptions on network connectivity and structure. In Section 3 we prove that these model equations have the qualitative properties observed in experimental settings. Important properties presented here include

- the existence of a globally attracting equilibrium solution,

- the shift of equilibria in response to both direct and indirect changes in value, and

- changes in the geometry of solutions with changes in value.

In Section 4 we manipulate the model equations to demonstrate a possible connection between normalization, the economic concept of time-discounting, and context-dependent choice behavior.

\section{Model Description}

Empirical and theoretical studies of normalization have focused largely on steady-state responses, producing a wide variety of functional forms to model neural systems at equilibrium. Dynamic models of normalization can explain temporal response characteristics unaddressed by static models $[1,3,11]$, but to date have not been applied to normalized value coding.

The dynamic model described in this paper assigns an output neuron $\left(R_{i}\right)$ and a gain control neuron $\left(G_{i}\right)$ to each available choice option $i$. Thus if there are $N$ options then the model is a system of $2 N$ differential equations. Each variable is a dimensionless measure of the firing rate of the corresponding neuron. We assume that at any given time, the greatest $R_{i}$ value determines choice of the subject, if a choice is forced at that time. Each gain control neuron computes a weighted sum of all of the output neurons in the network and inhibits its output neuron partner via divisive scaling (Figure 2). To model the time-varying activity of this network, we assume that

- the rate of change of the firing rate of each gain control neuron $G_{i}$ is proportional to the difference between its current firing rate and a weighted average of exponentiated firing rates for the response neurons and

- the rate of change of the firing rate of each output neuron $R_{i}$ is proportional to the difference between its current firing rate and the normalized pool rate via the gain control neurons.

These assumptions give the non-dimensional system of $2 N$ differential equations

$$
\begin{aligned}
& \tau \dot{G}_{i}=-G_{i}+\sum_{j=1}^{N} \omega_{i j} R_{j}^{n} \\
& \tau \dot{R}_{i}=-R_{i}+\frac{V_{i}}{1+G_{i}}
\end{aligned}
$$

where $i=1, \ldots, N$ corresponds to individual choice options. The most important parameters in the model are $V_{1}, \ldots, V_{N}$ which represent the intrinsic value associated with choice $i$, and thus we assume that $V_{i}>0$ for all $i$. The parameters $\omega_{i j} \geq 0$ weight the input from $R_{j}$ to the gain neuron $G_{i}$, and $\tau>0$ is an intrinsic timescale parameter. Finally, note that the parameter $n$ is greater than or equal to 1 . In non-dynamic normalization models, the value of this parameter is determined experimentally. For a discussion on the possible physiological significance of this parameter and its implications for decision-making see [10].

Previous work [6] has demonstrated that value coding is computed relative to the value of alternative options (via divisive normalization) when the time-dependent features of 


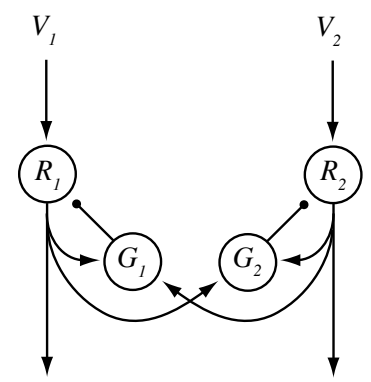

Figure 2: Proposed network for divisive normalization in a two-option setting. The parameters $V_{i}$ represent the intrinsic value of choice $i$. This is input into the response neuron $R_{i}$. The gain-control neurons $G_{i}$ aggregate the firing rates of all of the response neurons in the network and then inhibit their partner response neuron via normalization.

decision-making are ignored (i.e., at equilibrium). In [7] we show that the dynamic model of Equations (1) qualitatively predict a variety of phenomenon observed in single cell recordings in the monkey LIP during value-related decision-making tasks, including the equilibrium behavior previously described (with $N=2$ ). We also show that in a single-option scenario $(N=1)$, the dynamic model predicts how the output firing rate changes over time in a single-reward saccade task, and how this time-course changes with the reward value (Figure 1). We concluded that work by describing how Equations (1) imply a type of context dependence that we term discounted normalization (see Section 4).

\section{$3 \quad$ Properties of Solutions}

In this section we present the properties of Equations (1) relating to both equilibrium and non-equilibrium behavior.

\subsection{Properties of a "Single Choice" Model}

To provide a basic result on which we build, we begin by considering the system of Equations (1) when $N=1$. Subscripts will be omitted when presenting these results, and these equations simplify to

$$
\begin{aligned}
\tau \dot{G} & =-G+\omega R^{n} \\
\tau \dot{R} & =-R+\frac{V}{1+G} .
\end{aligned}
$$

Let $Q=\{(G, R): G \geq 0$ and $R \geq 0\}$.

Proposition 1. The equations in (2) have a unique, asymptotically stable equilibrium point $(\bar{G}, \bar{R}) \in Q$. Moreover, any solution $\phi_{t}\left(G_{0}, R_{0}\right)$ with $\left(G_{0}, R_{0}\right) \in Q$ satisfies

$$
\lim _{t \rightarrow \infty} \phi_{t}\left(G_{0}, R_{0}\right)=(\bar{G}, \bar{R}) .
$$

Proof. Without loss of generality, assume that $\tau=1$. The $G$-nullcline is given by $R=$ $(G / \omega)^{1 / n}$ and the $R$-nullcline by $R=V /(1+G)$. Both are continuous in the region $Q$. The $G$-nullcline passes through $(0,0)$ and is increasing. The $R$-nullcline passes through $(0, V)$, is decreasing, and limits on the $G$-axis as $G \rightarrow \infty$. Hence these curves have a unique intersection at an equilibrium point $(\bar{G}, \bar{R})$. 


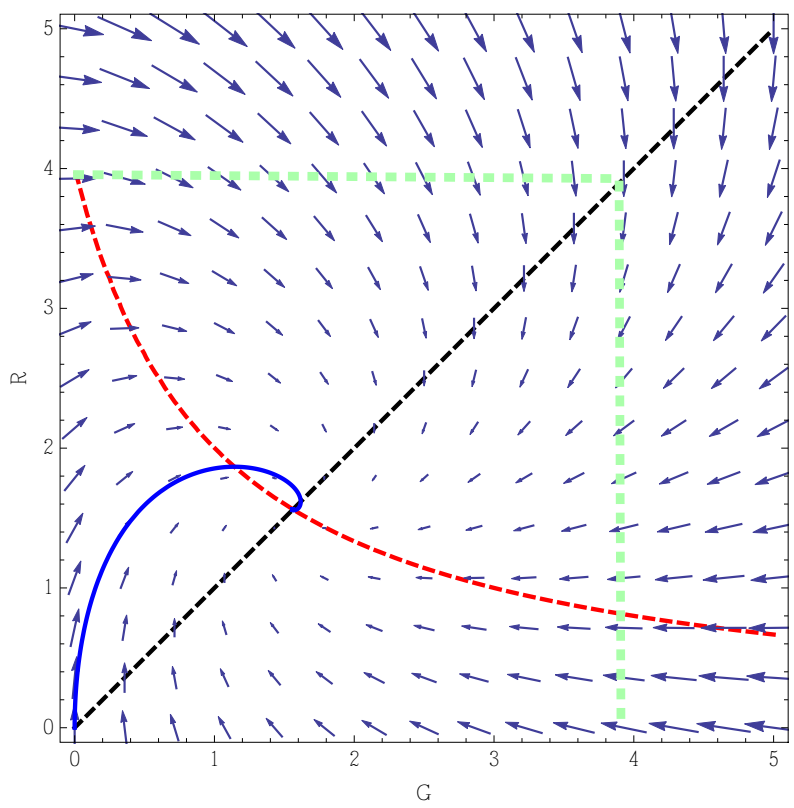

Figure 3: The phase plane of (2) with $N=1, \omega=1$, and $V=4$. The $G$-nullcline is the black, dashed line. The $R$-nullcline is the red, dashed line. The solution curve with $(G(0), R(0))=(0,0)$ is shown in blue. The region $Q^{\prime}(\rho)$ with $\rho=4$ is bounded by the coordinate axes and the green dashed lines.

The Jacobian matrix $J$ of Equations $(1)$ at $(\bar{G}, \bar{R})$ has both a negative trace and a positive determinant. Thus $(\bar{G}, \bar{R})$ is hyperbolic and asymptotically stable.

Define the region $Q^{\prime}(\rho)$ (see Figure 3 ) by

$$
Q^{\prime}(\rho)=\left\{(G, R) \in Q: R \leq \rho \text { and } G \leq \omega \rho^{n}\right\} .
$$

It is easy to show that for all $\rho \geq V$ the compact rectangular region $Q^{\prime}(\rho)$ is positively invariant. This implies that for all $\rho \geq V$ solutions with initial conditions in $Q^{\prime}(\rho)$ exist for all $t>0$ and thus these solutions have nontrivial $\omega$-limit sets. Moreover, since

$$
\operatorname{div}\left((\dot{G}, \dot{R})^{T}\right)=\nabla \cdot(\dot{G}, \dot{R})^{T}=\operatorname{Tr} J<0
$$

for all $G \geq 0, R \geq 0$ it follows from Bendixon's Criterion that there are no periodic orbits in any of these regions. Thus all solutions limit on the equilibrium $(\bar{G}, \bar{R})$.

Let $\pi_{R}: \mathbf{R}^{2} \rightarrow \mathbf{R}$ denote projection onto the $R$ coordinate.

Proposition 2. Consider Equations (2) and suppose that $G_{0}<\bar{G}$ and $R_{0}<\bar{R}$. Then $\pi_{R} \circ \phi_{t}\left(G_{0}, R_{0}\right)$ has a local maximum.

Proof. A solution $\phi_{t}\left(G_{0}, R_{0}\right)$ satisfying the given initial conditions must exit the rectangle $0<G_{0}<\bar{G}, 0<R_{0}<\bar{R}$ through the horizontal line $R=\bar{R}$ and then cross the $R$-nullcline at a value of $G<\bar{G}$. This corresponds to a local maximum.

Changes in Parameters A key question in almost all neuroeconomic research is how choice behavior changes with variations in the values of the choice set. Thus it is essential to understand how solution curves change as the value parameter $V$ varies. Figure 4 illustrates changes to the phase plane in response to changes in the parameter $V$. In particular, we show that as $V$ increases both the equilibrium and maximum firing rates increase. To simplify notation let $R(t ; V)=\pi_{R} \circ \phi_{t}\left(G_{0}, R_{0} ; V\right)$. 


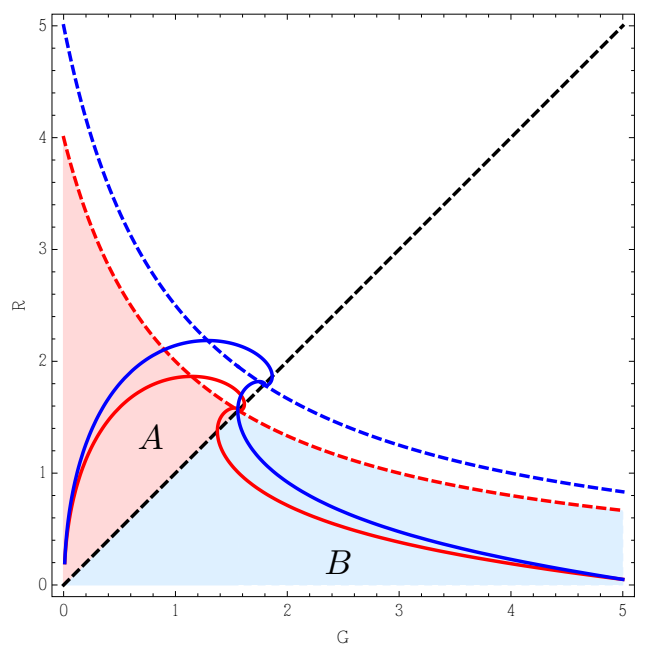

Figure 4: Graphs of the $G$ and $R$ nullclines (dashed), corresponding solution curves (solid), and the regions $A$ (light red) and $B$ (light blue) for two different values of $V$. The red curves correspond to $V=4$ while the blue to $V=5$. For each parameter value, we show solution curves with initial conditions in both $A$ and $B$.

Proposition 3. Consider the family of differential equations (2) depending on the parameter $V>0$.

1. The $R$-coordinate of the equilibrium point, $\bar{R}(V)$, is increasing.

2. If $V_{1}<V_{2}$ and $\left(G_{0}, R_{0}\right) \in\left[0, \bar{G}\left(V_{1}\right)\right) \times\left[0, \bar{R}\left(V_{1}\right)\right)$ then

$$
R\left(t_{1}^{*}, G_{0}, R_{0} ; V_{1}\right)<R\left(t_{2}^{*}, G_{0}, R_{0} ; V_{2}\right)
$$

where $t_{k}^{*}$ is the minimal $t>0$ such that $R\left(t_{k}^{*} ; V_{k}\right)$ is a local maximum.

Proof. Statement 1 follows immediately from the fact that in the region $Q$, the $R$-nullcline increases with $V$ and the $G$-nullcline is an increasing function of $R$.

Let $A_{V}$ denote the region bounded by the $R$-axis and the $G$ and $R$ nullclines (not including either nullcline) and $B_{V}$ the unbounded region defined by the $G$-axis, the $G$ nullcline and the $R$-nullcline for a given value of $V$. The slope of each vector defined by the vector field (2) is given by

$$
T=\frac{d R / d t}{d G / d t}=\frac{-R(1+G)+V}{(1+G)\left(-G+\omega R^{n}\right)} .
$$

The derivative $\partial T / \partial V$ describes the change in these slopes with respect to a change in the parameter $V$. If $(G, R) \in A_{V}$ then $\frac{\partial T}{\partial V}>0$ and thus the slopes of the vectors in this region increase with $V$ (see Figure 4). On the other hand, at points in the region $B_{V}$, slopes decrease with $V$.

Let $V_{1}<V_{2}$ and let $A=A_{V_{1}} \cap A_{V_{2}}$ and $B=B_{V_{1}} \cap B_{V_{2}}$. Each vector in $A$ is directed upward and to the right and each vector in $B$ is directed upward and to the left.

Let's first consider the region $A$. Since $\frac{\partial T}{\partial V}>0$ in $A$, at each point in $A$ the vector corresponding to $V_{2}$ is steeper than the vector based at the same point defined by $V_{1}$. This implies that for $\left(G_{0}, R_{0}\right) \in A, t>0$ and both $\phi_{t}\left(G_{0}, R_{0} ; V_{1}\right)$ and $\phi_{t}\left(G_{0}, R_{0} ; V_{2}\right)$ in $A$

$$
\pi_{G} \circ \phi_{t}\left(G_{0}, R_{0} ; V_{2}\right)<\pi_{G} \circ \phi_{t}\left(G_{0}, R_{0} ; V_{1}\right) .
$$

Since $\phi_{t}\left(G_{0}, R_{0} ; V_{2}\right)$ lies to the left of $\phi_{t}\left(G_{0}, R_{0} ; V_{1}\right)$ in the region $A$, it follows that the trajectory $\phi_{t}\left(G_{0}, R_{0} ; V_{2}\right)$ intersects the graph of $R=V_{2} /(1+G)$ at a smaller value of $G$ (call it 
$G_{2}^{*}$ and the corresponding time $\left.t_{2}^{*}\right)$ than the corresponding intersection of $\phi_{t}\left(G_{0}, R_{0} ; V_{1}\right)$ and the graph of $R=V_{1} /(1+G)$ (call it $G_{1}^{*}$ and the corresponding time $t_{1}^{*}$ ). Since $R=V /(1+G)$ is a decreasing function of $G$ and $\frac{\partial}{\partial V} \frac{V}{1+G}>0$ it follows that

$$
R\left(t_{1}^{*}, G_{0}, R_{0} ; V_{1}\right)<R\left(t_{2}^{*}, G_{0}, R_{0} ; V_{2}\right)
$$

for $\left(G_{0}, R_{0}\right) \in A$.

If $\left(G_{0}, R_{0}\right) \in B$ then a similar argument shows that

$$
\pi_{R} \circ \phi_{t}\left(G_{0}, R_{0} ; V_{2}\right)<\pi_{R} \circ \phi_{t}\left(G_{0}, R_{0} ; V_{1}\right)
$$

in $B$. Hence $\phi_{t}\left(G_{0}, R_{0} ; V_{2}\right)$ intersects the $G$-nullcline at a smaller value of $G$ than the corresponding intersection of $\phi_{t}\left(G_{0}, R_{0} ; V_{1}\right)$ with the $G$-nullcline. A second argument, almost identical to the one above, implies that

$$
R\left(t_{1}^{*}, G_{0}, R_{0} ; V_{1}\right)<R\left(t_{2}^{*}, G_{0}, R_{0} ; V_{2}\right)
$$

for $\left(G_{0}, R_{0}\right) \in B$.

\subsection{Properties of a Multiple Choice Model}

We now turn our consideration to general systems of differential equations based on those studied above. Let $\mathcal{G}=\left(G_{1}, G_{2}, \ldots, G_{N}\right)^{T} \in \mathbf{R}^{N}$ and $\mathcal{R}=\left(R_{1}, R_{2}, \ldots, R_{N}\right)^{T} \in \mathbf{R}^{N}$ and rewrite Equations (1) as

$$
\begin{aligned}
\dot{\mathcal{G}} & =-\mathcal{G}+W \mathcal{R}^{n} \\
\dot{\mathcal{R}} & =-\mathcal{R}+V(\mathcal{G})
\end{aligned}
$$

where the $N \times N$ matrix $W=\left[\omega_{i j}\right], \mathcal{R}^{n}=\left(R_{1}^{n}, R_{2}^{n}, \ldots, R_{N}^{n}\right)^{T}$ and

$$
V(\mathcal{G})=\left(\frac{V_{1}}{1+G_{1}}, \frac{V_{2}}{1+G_{2}}, \ldots, \frac{V_{N}}{1+G_{N}}\right)^{T} .
$$

Define the diagonal matrix $W_{d}=\left[\omega_{i j} \delta_{i j}\right]$ where

$$
\delta_{i j}=\left\{\begin{array}{cc}
1 & \text { if } i=j \\
0 & \text { otherwise }
\end{array} .\right.
$$

Proposition 4. If $\left\|W-W_{d}\right\|$ is sufficiently small then System (3) has a unique asymptotically stable equilibrium in $Q^{N}$.

Proof. When $W=W_{d}$ then Equations (3) decouple to a system of $N$ pairs of differential equations each satisfying the hypotheses of Proposition 1. Each pair of equations has a unique, hyperbolic, asymptotically stable equilibrium point $\left(\bar{G}_{i}, \bar{R}_{i}\right) \in Q$ for $i=1$ to $N$. Thus in this case Equations (3) also have a unique asymptotically stable equilibrium at $(\overline{\mathcal{G}}, \overline{\mathcal{R}})$ where $\overline{\mathcal{G}}=\left(\bar{G}_{1}, \ldots, \bar{G}_{N}\right)$ and $\overline{\mathcal{R}}=\left(\bar{R}_{1}, \ldots, \bar{R}_{N}\right)$. Because $(\overline{\mathcal{G}}, \overline{\mathcal{R}})$ is structurally stable, it follows immediately that Equations (3) also have a unique asymptotically stable equilibrium in some neighborhood of $(\overline{\mathcal{G}}, \overline{\mathcal{R}})$ for $\left\|W-W_{d}\right\|$ is sufficiently small.

In Proposition 3 we showed that in the single pair model the equilibrium firing rate $\bar{R}$ increases with $V$. In the following propositions we show that this property persists in certain multiple pair models. We also show that the equilibrium firing rate response of unit $i$ decreases as the parameter $V_{j}$ increases (with $i \neq j$ ) in these models. These properties are important partially because they provide one avenue for experimentally validating a normalization model.

Recall that the equilibria are given by the system of $N$ equations

$$
0=-R_{i}+V_{i}\left(1+\sum_{j=1}^{N} \omega_{i j} R_{j}^{n}\right)^{-1}:=F_{i}\left(R_{1}, \ldots, R_{n}, V_{1}, \ldots, V_{n}\right), i=1, \ldots N
$$


Let $F=\left(F_{1}, \ldots, F_{n}\right)$ and $\partial F / \partial \mathcal{R}, \partial F / \partial V$ denote the $N \times N$ matrices of partial derivatives with respect to $\mathcal{R}$ and $V=\left(V_{1}, \ldots, V_{N}\right)$ respectively.

Proposition 5. If $N=2,\left\|W-W_{d}\right\|$ is sufficiently small, and $\operatorname{det}(\partial F / \partial \mathcal{R})>0$, then the equilibria of Equations (4) satisfy

$$
\frac{\partial \overline{R_{i}}}{\partial V_{i}} \geq 0 \text { and } \frac{\partial \overline{R_{i}}}{\partial V_{j}} \leq 0, i \neq j .
$$

Proof. From Proposition 4 we know that there exists a nontrivial solution to Equation (4) if $\left\|W-W_{d}\right\|$ is sufficiently small. Let $\partial R / \partial V$ denote the $2 \times 2$ matrix of partial derivatives of the equilibrium firing rates with respect to the parameters $V_{i}$. It follows from the Implicit Function Theorem that

$$
\frac{\partial R}{\partial V}=-\left[\frac{\partial F}{\partial \mathcal{R}}\right]^{-1} \frac{\partial F}{\partial V}=\frac{-1}{\operatorname{det}(\partial F / \partial \mathcal{R})}\left(\begin{array}{cc}
\frac{\partial F_{2}}{\partial R_{2}} \frac{\partial F_{1}}{\partial V_{1}} & -\frac{\partial F_{1}}{\partial R_{2}} \frac{\partial F_{2}}{\partial V_{2}} \\
\frac{-\partial F_{2}}{\partial R_{1}} \frac{\partial F_{1}}{\partial V_{1}} & -\frac{\partial F_{1}}{\partial R_{1}} \frac{\partial F_{2}}{\partial V_{2}}
\end{array}\right) .
$$

By differentiating Equations (4) directly it is easy to see that $\partial F_{i} / \partial R_{j}<0$ for all $i$ and $j$ since $n>1$. In addition, $\partial F_{i} / \partial V_{i}>0$ for all $i$. Thus the entries in each row of $\partial R / \partial V$ are of opposite sign. Inequalities (5) then follow directly from the given determinant condition.

The proof of Proposition 5 depends critically on our ability to explicitly compute $\left(\frac{\partial F}{\partial \mathcal{R}}\right)^{-1}$ which was possible because there is a simple formula for inverting $2 \times 2$ matrices. But there is no such general formula for arbitrary invertible $N \times N$ matrices. However, in the special, but relevant, case where $\omega_{i j}=\omega$ for all $i$ and $j$ and the exponent $n=1$, we can find such a formula using the following lemma. Its straightforward proof is not presented here.

Lemma 1. Let $C$ be an $n \times n$ matrix with identical columns $\vec{c}=\left(c_{1}, \ldots, c_{n}\right)^{T}$ and define $M=I+C$. Then $\operatorname{det} M=1+\gamma$ where $\gamma=\sum_{k=1}^{n} c_{k}$. In addition, if $\gamma \neq-1$ then

$$
M^{-1}=I-\frac{1}{1+\gamma} C \text {. }
$$

Proposition 6. If $n=1, \omega_{i j}=\omega$ for all $i$ and $j$ and $\left\|W-W_{d}\right\|$ is sufficiently small then there exists nontrivial solutions to the equilibria equations (4) that satisfy

$$
\frac{\partial \overline{R_{i}}}{\partial V_{i}} \geq 0 \text { and } \frac{\partial \overline{R_{i}}}{\partial V_{j}} \leq 0, i \neq j .
$$

Proof. As before, Proposition 4 implies that there exists a nontrivial solution to Equations (4) if $\left\|W-W_{d}\right\|$ is sufficiently small. Because $n=1$ and $\omega_{i j}=\omega$ for all $i, j$ the Jacobian matrix $\frac{\partial F}{\partial \mathcal{R}}$ is given by

$$
\frac{\partial F}{\partial \mathcal{R}}=-(I+\alpha \mathcal{V})
$$

where

$$
\mathcal{V}=\left(\begin{array}{ccc}
V_{1} & \ldots & V_{1} \\
V_{2} & \ldots & V_{2} \\
\vdots & \ldots & \vdots \\
V_{N} & \ldots & V_{N}
\end{array}\right)
$$

and

$$
\alpha=\frac{\omega}{\left(1+\omega \sum_{i=1}^{N} R_{i}\right)^{2}}>0 .
$$

Applying Lemma 1 we get

$$
\left[\frac{\partial F}{\partial \mathcal{R}}\right]^{-1}=\left(\frac{\alpha}{1+\alpha N \sum_{j=1}^{N} V_{j}}\right) \mathcal{V}-I
$$


Substituting this into

$$
\frac{\partial R}{\partial V}=-\left[\frac{\partial F}{\partial \mathcal{R}}\right]^{-1} \frac{\partial F}{\partial V}
$$

and expanding the result we see that the diagonal entries satisfy

$$
\frac{\partial R_{i}}{\partial V_{i}}=\left(1-\frac{\alpha V_{i}}{1+\alpha N \sum_{j=1}^{N} V_{j}}\right) \frac{\partial F_{i}}{\partial V_{i}} \geq 0
$$

since the fraction in parentheses is between 0 and 1 . Similarly, the non-diagonal entries satisfy

$$
\frac{\partial R_{i}}{\partial V_{j}}=-\left(\frac{\alpha V_{i}}{1+\alpha N \sum_{j=1}^{N} V_{j}}\right) \frac{\partial F_{i}}{\partial V_{i}} \leq 0
$$

proving the result.

\section{Discounting}

In this section, we show that Equations (1) can be rewritten in alternative forms, suggesting that the neural mechanism described here can account for important context-dependent phenomena observed empirically. We refer to these forms as the discounted normalization equations and present both continuous and discrete versions of these equations.

To derive the continuous discounted normalization equation from (2) we begin by rewriting the first equation as

$$
\dot{G}+\frac{1}{\tau} G=\frac{\omega}{\tau} R(t)^{n} .
$$

If we now multiply both sides of this equation by $e^{t / \tau}$ and then apply the product rule we get

$$
\frac{d}{d t}\left(e^{\frac{t}{\tau}} G\right)=\frac{\omega}{\tau} e^{\frac{t}{\tau}} R(t)^{n}
$$

Integrating both sides of this equation and assuming that $G(0)=G_{0} \geq 0$ yields

$$
G(t)=G_{0} e^{-t / \tau}+\frac{\omega}{\tau} \int_{0}^{t} e^{-\frac{1}{\tau}(t-s)} R(s)^{n} d s=G_{0} e^{-t / \tau}+\frac{\omega}{\tau} E(t) * R(t)^{n} .
$$

where $E(t)=e^{-t / \tau}$ and $*$ denotes convolution. Substituting this expression into the second equation of (2) yields the continuous discounted normalization equation

$$
\tau \dot{R}=-R+V\left(1+G_{0} e^{-t / \tau}+\frac{\omega}{\tau}\left[E * R^{n}\right](t)\right)^{-1} .
$$

A form more amenable to interpretation can be derived by first discretizing Equations (2) via Euler's method with a step size of $h \tau$ with $0<h<1$. More precisely, let $t$ be an integer and use the approximations

$$
\dot{G} \approx \frac{G(t+1)-G(t)}{h \tau} \quad \text { and } \quad \dot{R} \approx \frac{R(t+1)-R(t)}{h \tau}
$$

for $h$ sufficiently small. This gives the discrete system

$$
\begin{aligned}
& G(t+1)=\alpha G(t)+\omega R(t) \\
& R(t+1)=\alpha R(t)+\frac{V}{1+G(t)}
\end{aligned}
$$


where $\alpha=1-h>0$ and the parameters $\omega$ and $V$ are rescaled from (2) by a factor of $h$. Assume initial conditions of $G(0)=G_{0} \geq 0$ and $R(0)>0$. It follows immediately that

$$
\begin{aligned}
& G(1)=\alpha G_{0}+\omega R(0)^{n} \\
& R(1)=\alpha R(0)+V .
\end{aligned}
$$

The next terms in the sequence are

$$
\begin{aligned}
& G(2)=\alpha^{2} G_{0}+\omega\left(\alpha R(0)^{n}+R(1)^{n}\right) \\
& R(2)=\alpha R(1)+\frac{V}{1+G(1)}
\end{aligned}
$$

By induction, we get the generalized $G$ term of

$$
G(t+1)=\alpha^{t+1} G_{0}+\omega \sum_{k=0}^{t} \alpha^{k} R(t-k)^{n} .
$$

Substituting this into the $R$ recurrence in (8) gives the discrete discounted normalization equation

$$
R(t+1)=\alpha R(t)+\frac{V}{1+\alpha^{t} G_{0}+\omega \sum_{k=0}^{t-1} \alpha^{k} R(t-k-1)^{n}}
$$

as desired.

The derivation of Equation (9) from (2) reveals an important implication of this dynamical normalization model: dynamic normalization inherently incorporates a discounted sum of firing rates at previous time steps with more temporally distant firing rates discounted more heavily. The parameter $\alpha$ in (9) can be interpreted as a discount rate in the normalization. It measures the impact of earlier firing rates on the normalization to the next firing rate $R(t+1)$. In particular, the firing rate $k$ time steps in the past is discounted by a factor of $\alpha^{k}$ in the normalizing summation. Thus the closer $\alpha$ is to 1 , the longer the "memory" in the normalization.

A similar discounted normalization formula is briefly presented in [2] but is not derived. In that paper Carandini and Heeger state that equations such as this can be applied when modeling "adaptation, such as light adaptation in retina or contrast adaptation in visual cortex." Thus significantly, our physiologically motivated differential equations model of normalization inherently implements a time-dependent normalization model that can be used to model adaptation in the visual system or similarly context-dependence in a decisionmaking setting.

These ideas generalize naturally for the system of Equations (1) modeling a multiple choice decision-making process. In this scenario the continuous discounted normalization equations become

$$
\tau \dot{R}_{i}=-R_{i}+V_{i}\left(1+G_{i}(0) e^{-t / \tau}+\frac{1}{\tau} \sum_{j=1}^{N} \omega_{i j} E(t) *\left(R_{j}(t)\right)^{n}\right)^{-1}
$$

and the discrete become

$$
R_{i}(t+1)=\alpha R_{i}(t)+\frac{V_{i}}{1+\alpha^{t} G_{i}(0)+\sum_{k=0}^{t-1} \sum_{j=1}^{N} \alpha^{k} \omega_{i j} R_{j}(t-k-1)^{n}} .
$$

These equations suggest that in a scenario with multiple options, the firing rate of a given response neuron is normalized both temporally and spatially. In other words, the firing rate depends on both the values of all of the items in the current choice set (via the corresponding firing rates) and a temporally discounted accounting of previous choice set values (again via firing rates). 


\section{Discussion}

The results presented in this paper are first steps in developing a dynamic theory of normalization in perceptual and decision-making neural circuits. As such, the results of Section 3 describe the fundamental properties of the model Equations (1). The properties described match empirical properties observed in electrophysiological recordings from decision-related brain areas.

Propositions 1 and 2 describe the time-course of firing rate curves in a single-option "choice" task and imply that all physically relevant solutions have a characteristic geometry; they increase from some baseline firing rate to a maximum firing rate and then decay towards an equilibrium firing rate before reward-delivery. In [7] we present the results of a single-option choice experiment that manipulated reward value $\left(V_{1}\right)$ and show the correspondence between these solution curves and actual firing rate curves. Of special interest is the existence of the local maximum prior to equilibrium, which produces the characteristic phasic transient in neural activity following option presentation. Our model suggests that these dynamics can arise entirely from the proposed normalization network structure and need not be inherited from dynamic variation of external inputs.

At its core, neuroeconomics is about understanding the neural mechanisms of decisionmaking that encode and compare the valuations of multiple choice alternatives with different values. Thus the results of Propositions 4 through 6 are central to this work. Proposition 4 implies that in multiple-option models, solution curves still approach an equilibrium solution. We have not proven that the $R_{i}$ components of these multi-element solution curves reach a maximum firing rate before decaying to equilibrium. However, numerical explorations of these systems suggest that they do retain this feature as well.

A key characteristic of normalization in cortical circuits is the dependence of firing rate on both direct and indirect input [2,4]. In particular, direct input (such as increasing the reward associated with an option in the response field of a neuron) should increase the the firing rate of that neuron while indirect input (increasing the reward associated with options outside the response field of the neuron under study) should decrease firing rate. Propositions 5 and 6 show that at equilibrium, the dynamic model captures this feature in two special, but reasonable, settings. In [7], we show that the model accurately characterizes experimental firing rates recorded in parietal cortex in response to both RF target and extra-RF target value changes.

Of special interest to us are the discounting results of Section 4. These result connect neural circuitry to fundamental economic concepts (expectations and reference dependence) and suggest a possible neural mechanism for context dependent choice behavior. Because the "discount rate" $\alpha$ lies between 0 and 1, these equations imply that the current firing rate of a neuron is normalized more heavily by recent firing rates than by earlier rates. This suggests that in a situation where multiple different options are presented sequentially, recent options more significantly influence firing rates than early options. Critically, this formulation introduces an intrinsic history-dependence into the neural representation of value. In the economic literature, reference-dependence is a widespread feature of human decision-making that deviates from strictly utility-based models of rational choice. Our neural-based normalization model suggests that some forms of apparently inconsistent choice behavior may arise from this underlying circuitry responsible for valuation and decisionmaking.

The implementation of the divisive normalization computation in a dynamical model has both theoretical and empirical implications. By providing a framework for time-varying information coding, the dynamic model extends the study of neural information processing from equilibrium activity to periods of phasic or transient activity. The existence of significant early value coding suggests an expanded focus for neurobiological studies of decision making, which primarily examine neural activity late in the decision process. Furthermore, our model links neural information coding to the architecture of the underlying neural circuit, in particular emphasizing the potential role of lateral inhibition in the dynamic 
computation of normalized contextual information. While little is currently known about the empirical role of inhibitory neurons in the decision process, the dynamic normalization model predicts a characteristic inhibition time course that invites further experimental study.

While this paper focuses on normalization in decision-related value coding, the dynamical model can easily be generalized to other neural processes that employ the normalization computation. Divisive normalization was originally proposed to explain nonlinearities in early visual processing, but has been demonstrated in multiple sensory modalities and levels of processing in numerous organisms, leading to the proposal that normalization is a canonical neural computation [2]. Most studies of sensory normalization processes emphasize the analysis of equilibrium levels of neural activity, though dynamical models show promise in explaining additional characteristics of such sensory coding $[1,3,11]$. Our model extends the dynamical approach to decision-related processing, but also suggests that a circuit architecture-based model of temporal activity holds promise for further understanding of normalization in sensory coding.

\section{References}

[1] Matteo Carandini and David J. Heeger. Summation and division by neurons in primate visual cortex. Science, 264(5163):1333, 1994.

[2] Matteo Carandini and David J. Heeger. Normalization as a canonical neural computation. Nat Rev Neurosci, 13:51-62, 2012.

[3] Matteo Carandini, David J. Heeger, and J. Anthony Movshon. Linearity and normalization in simple cells of the macaque primary visual cortex. The Journal of Neuroscience, 17(21):8621-8644, 1997.

[4] David J. Heeger. Normalization of cell responses in cat striate cortex. Visual Neuroscience, 9:181-197, 1992.

[5] Kenway Louie and Paul W. Glimcher. Separating value from choice: delay discounting activity in the lateral intraparietal area. The Journal of Neuroscience, 30(16):5498$5507,2010$.

[6] Kenway Louie, Lauren E. Grattan, and Paul W. Glimcher. Reward value-based gain control: Divisive normalization in parietal cortex. Journal of Neuroscience, 31(29):10627-10639, 2011.

[7] Kenway Louie, Thomas LoFaro, Ryan Webb, and Paul W. Glimcher. A dynamic normalization model predicts time-varying value coding in decision-related circuits. Journal of Neuroscience, in press, 2014.

[8] Michael L. Platt and Paul W Glimcher. Neural correlates of decision variables in parietal cortex. Nature, 400(6741):233-238, 1999.

[9] Leo P. Sugrue, Greg S. Corrado, and William T. Newsome. Matching behavior and the representation of value in the parietal cortex. Science, 304(5678):1782-1787, 2004.

[10] Ryan Webb, Paul W. Glimcher, and Kenway Louie. Rationalizing context-dependent preferences: Divisive normalization and neurobiological constraints on decision-making. Under Review, 2014.

[11] Hugh R. Wilson and Richard Humanski. Spatial frequency adaptation and contrast gain control. Vision Research, 33(8):1133-1149, 1993.

[12] Wolfram Research. Mathematica, volume 10. Wolfram Research, Inc., 2014. 